\title{
The $\kappa-\mu$ Shadowed Fading Model with Arbitrary Intercluster Correlation
}

\author{
Pablo Ramírez-Espinosa, José F. Paris, José A. Cortés and Eduardo Martos-Naya \\ Dpto. de Ingeniería de Comunicaciones, ETSI Telecomunicacion, Universidad de Málaga, Málaga, E-29071, Spain \\ email: \{pre, paris, jaca, eduardo\}@ic.uma.es
}

\begin{abstract}
In this paper, we propose a generalization of the well-known $\kappa-\mu$ shadowed fading model. Based on the clustering of multipath waves as the baseline model, the novelty of this new distribution is the addition of an arbitrary correlation for the scattered components within each cluster. It also inherits the random fluctuation of the dominant component, which is assumed to be the same for all clusters. Thus, it unifies a wide variety of models: Rayleigh, Rician, Rician shadowed, Nakagami$m, \kappa-\mu$ and $\kappa-\mu$ shadowed as well as multivariate Rayleigh, Rician and Rician shadowed. The main statistics of the newly proposed model, i.e. moment generating function, probability density function and cumulative density function, are given in terms of exponentials and powers, and some numerical results are provided in order to analyze the impact of the arbitrary intercluster correlation.
\end{abstract}

\section{INTRODUCTION}

The statistical characterization of the received radio signal is a classical problem in wireless communications, where the transmitted radio waves are affected by several random phenomena. As a result, the received signal can be seen as a linear combination of multipath waves with random amplitudes and phases. By applying the central limit theorem, the baseband signal is modeled using complex Gaussian processes, rendering the widely-used fading models, namely Rayleigh, Rice, Hoyt and Nakagami-m [1].

In order to provide a better statistical characterization in intricate multipath environments, several distributions have been proposed in the literature as generalizations of the classical ones. In [2], Yacoub introduces two new models based on the effect of clustering of multipath waves: the $\eta$ - $\mu$ fading model as a generalization of the Hoyt model and the $\kappa$ - $\mu$ fading model, which arises as a generalization of the Rician model. Due to the small number of parameters along with the comparatively simple mathematical tractability, a lot of attention has been paid to the $\kappa-\mu$ model in the last years [3-5]. Although a more general model was introduced in [6], its larger flexibility comes at the price of an increased mathematical complexity, ultimately affecting its practical applicability.

A further generalization of the $\kappa-\mu$ distribution was presented in [7] where the line of sight (LoS) component is affected by a random fluctuation representing the shadowing,

This work has been funded by the Spanish Government and the European Fund for Regional Development FEDER (project TEC2014-57901-R) and also by the Campus de Excelencia Internacional Andalucía TECH, Universidad de Málaga. rendering the well-known $\kappa-\mu$ shadowed model. This fading distribution has applications in mobile satellite (LMS) communications and underwater acoustic communications (UAC), including a wide variety of models as special cases: Rayleigh, Rice, Rician shadowed [8], Nakagami- $m, \kappa-\mu$ and Hoyt.

However, all of the aforementioned fading models assume the statistical independence between the radio signal components of all clusters. Attempting to consider the effect of the correlation, Bhatnagar characterizes in [9] the sum of $\kappa-\mu$ shadowed variables with correlated LoS fluctuation, providing the PDF in terms of infinite series. Nonetheless, the scattered components are still mutually uncorrelated.

In order to account for the impact of such intercluster cross correlation, we here introduce a novel extension of the $\kappa-\mu$ shadowed fading model which considers an arbitrary correlation between the scattered components. Thus, we consider appropriate to name this new distribution as correlated $\kappa$ $\mu$ shadowed distribution. Aiming to relay the mathematical complexity of the newly proposed fading model, we take the formulation of the $\kappa-\mu$ shadowed model with integer parameters as starting point [10]. Interestingly, despite being more general than the original $\kappa-\mu$ shadowed distribution, this new model inherits its simple mathematical tractability. Hence, the main statistics of the proposed model: Moment Generating Function (MGF), Probability Density Function (PDF) and Cumulative Distribution Function (CDF) are given in simple closed-form involving only elementary functions (exponentials and powers). Notably, in contrast to the original one, the correlated $\kappa-\mu$ shadowed fading model also includes as particular cases the multivariate Rayleigh, Rician and Rician shadowed distributions [11-14].

Throughout this paper, we will use the following notation. The symbol $\sim$ means statistically distributed as. Matrices and vectors are represented with upper-case boldface and lowercase boldface, respectively. The superscript $(\cdot)^{\dagger}$ denotes matrix complex conjugate transpose. Additionally, $\mathbf{I}_{n}$ represents the $n \times n$ identity matrix and $\mathbf{0}_{n \times p}$ a $n \times p$ all-zero matrix. Finally, $(a)_{b}$ is the Pochhammer symbol and $\mathbb{E}[\cdot]$ is the expectation operator.

This paper is structured as follows. In Section II, we describe the physical model of the proposed fading distribution. Then, the first order statistics (MGF, PDF and CDF) are derived in Section III, while we provide some numerical results in Section IV to exemplify the impact of the distribution 
parameters. Finally, main conclusions are given in Section V.

\section{PhySiCAL MODEL}

The physical model of the correlated $\kappa-\mu$ shadowed fading distribution arises as a generalization of the original one in [7]. The received radio signal is modeled as the superposition of radio waves structured in clusters of waves, where we consider that the scattered component has the same power within each cluster. The same applies to the dominant components, which are assumed to have equal power for all clusters. As opposed to the $\kappa-\mu$ shadowed distribution, we here introduce an arbitrary correlation factor for the intercluster scattered components. Thus, the received signal power $W$ can be formulated using complex random variables as

$$
W=\sum_{i=1}^{\mu}\left|Z_{i}+\xi p\right|^{2}
$$

where $\mu$ is a natural number indicating the number of clusters and $Z_{i}$ for $i=1, \ldots, \mu$ are complex Gaussian random variables such that $Z_{i} \sim \mathcal{C N}\left(0, \sigma^{2}\right)$. Additionally, $p$ is a complex number and $\xi$ is a real random variable such that $\xi^{2}$ is Gamma distributed with shape parameter $m$ and scale parameter $1 / m$, i.e. $\xi^{2} \sim \Gamma(m, 1 / m)$ with $\mathbb{E}\left[\xi^{2}\right]=1$. As with the original $\kappa-\mu$ shadowed model, $\xi$ represents the random fluctuation of the dominant component of all clusters of waves. The distinct variables $Z_{i}$ in (1) are assumed to be correlated with correlation factor given by $\operatorname{corr}\left(Z_{i}, Z_{j}\right)=\rho_{i, j}$ for $i, j=1, \ldots, \mu$ with

$$
\rho_{i, j}=\frac{\mathbb{E}\left[Z_{i} Z_{j}^{*}\right]}{\sigma^{2}} .
$$

Therefore, the novelty of the model here introduced is the addition of such arbitrary intercluster correlation factor. Note that, if we impose $\rho_{i, j}=0 \forall i \neq j$, then (1) becomes the $\kappa-\mu$ shadowed physical model in [7, eq. (1)]. Since this correlation implies a considerable difficulty in the statistical characterization of the physical model in (1), we will reformulate $W$ in terms of statistically independent random variables as follows.

The summation in (1) can be written in matrix form as a non-central complex Gaussian quadratic form as

$$
W=(\mathbf{z}+\xi \mathbf{p})^{\dagger}(\mathbf{z}+\xi \mathbf{p})
$$

where $\mathbf{z} \in \mathbb{C}^{\mu \times 1}$ and $\mathbf{p} \in \mathbb{C}^{\mu \times 1}$ are column vectors whose entries are $Z_{i}$ and $p$ for $i=1, \ldots, \mu$, respectively. As such, $\mathbf{z}$ follows a complex multivariate normal distribution with zero mean and correlation matrix $\boldsymbol{\Sigma}$ with entries $[\boldsymbol{\Sigma}]_{i, j}=\rho_{i, j}$, i.e. $\mathbf{z} \sim \mathcal{C N}_{\mu}\left(\mathbf{0}_{\mu \times 1}, \sigma^{2} \boldsymbol{\Sigma}\right)$. The correlation matrix $\boldsymbol{\Sigma}$ is Hermitian, so it can be decomposed as $\boldsymbol{\Sigma}=\mathbf{C C}^{\dagger}$ where $\mathbf{C} \in \mathbb{C}^{\mu \times \mu}$ is a lower triangular matrix with non-negative diagonal entries (Cholesky decomposition)[15].

Then, $\mathbf{z}$ can be expressed as $\mathbf{z}=\sigma \mathbf{C} \widetilde{\mathbf{z}}$, with $\widetilde{\mathbf{z}}$ a standard complex multivariate Gaussian vector, i.e. $\widetilde{\mathbf{z}} \sim$ $\mathcal{C N}_{\mu}\left(\mathbf{0}_{\mu \times 1}, \mathbf{I}_{\mu}\right)$. By doing so, (3) is rewritten as

$$
W=\left(\sigma \widetilde{\mathbf{z}}+\xi \mathbf{C}^{-1} \mathbf{p}\right)^{\dagger} \mathbf{C}^{\dagger} \mathbf{C}\left(\sigma \widetilde{\mathbf{z}}+\xi \mathbf{C}^{-1} \mathbf{p}\right) .
$$

Since $\mathbf{C}^{\dagger} \mathbf{C}$ is clearly Hermitian, it can be diagonalized as $\mathbf{C}^{\dagger} \mathbf{C}=\mathbf{U} \boldsymbol{\Lambda} \mathbf{U}^{\dagger}$, where $\boldsymbol{\Lambda}$ is a diagonal matrix whose entries, $\lambda_{i}$, are the eigenvalues of $\mathbf{C}^{\dagger} \mathbf{C}$ (or, equivalently, those of $\boldsymbol{\Sigma}$ ) and $\mathbf{U}$ is an unitary matrix whose $i$-th column is the eigenvector associated with $\lambda_{i}$. Therefore, (4) is finally expressed in a similar way to (1) as

$$
W=\sum_{i=1}^{\mu}\left|\sqrt{\lambda_{i}} \sigma \widetilde{Z}_{i}+\xi \sqrt{\lambda_{i}} \widetilde{p}_{i}\right|^{2}
$$

where $Z_{i}$ are statistically independent complex Gaussian random processes with zero mean and unit variance, i.e. $Z_{i} \sim$ $\mathcal{C N}(0,1)$, and $\widetilde{p}_{i}$ are the entries of the vector $\widetilde{\mathbf{p}}=\mathbf{U}^{\dagger} \mathbf{C}^{-1} \mathbf{p}$ for $i=1, \ldots, \mu$.

Thus, we have proved that the physical model in (1) is equivalent to another one where all the involved random variables are independent. Consequently, the power of each Gaussian variable $Z_{i}$ in (5) is rescaled by the eigenvalues of $\boldsymbol{\Sigma}$ and, in contrast with (1), the power of the dominant component is not the same for all clusters. This new representation of the physical model in terms of independent random variables will lead us to simple expressions of the main statistics (MGF, PDF and $\mathrm{CDF}$ ) of the here proposed model.

\section{StatisticAl CharacterizATION}

We will now provide the first-order statistics of the correlated $\kappa-\mu$ shadowed distribution where its MGF, PDF and CDF will be given in closed-form in terms of elementary functions. Hence, as usually done in the related literature, hereinafter we will consider the random variable $\gamma \triangleq \bar{\gamma} W / \bar{W}$ representing the instantaneous SNR at the receiver side, where $\bar{\gamma} \triangleq \mathbb{E}[\gamma]$ and $\bar{W}=\mathbb{E}[W]=\mu\left(|p|^{2}+\sigma^{2}\right)$.

Therefore, let $\gamma$ be a random variable characterizing the instantaneous SNR for the physical model in (1) or, equivalently, that in (5). Then, $\gamma$ follows a correlated $\kappa$ - $\mu$ shadowed distribution with parameters $\mu, m, \kappa$ and $\Sigma$, i.e. $\gamma \sim$ $\mathcal{C S}_{\kappa \mu}(\bar{\gamma} ; \kappa, \mu, m, \boldsymbol{\Sigma})$ with $\kappa=|p|^{2} / \sigma^{2}$.

Lemma 1: Let $\gamma \sim \mathcal{C S}_{\kappa \mu}(\bar{\gamma} ; \kappa, \mu, m, \boldsymbol{\Sigma})$. Then, its MGF is given by

$$
\begin{aligned}
M_{\gamma}(s)= & \frac{\mu^{\mu}(\kappa+1)^{\mu}}{\prod_{i=1}^{\mu} \mu(\kappa+1)-s \lambda_{i} \bar{\gamma}} \\
& \times\left(1-\frac{1}{m} \sum_{j=1}^{\mu} \frac{d_{i} \lambda_{i} s \bar{\gamma}}{\mu(\kappa+1)-s \lambda_{i} \bar{\gamma}}\right)^{-m}
\end{aligned}
$$

where $d_{i}=\left|\widetilde{p}_{i}\right|^{2} / \sigma^{2}=\left[\mathbf{U}^{\dagger} \mathbf{C}^{-1} \mathbf{K}\left(\mathbf{C}^{-1}\right)^{\dagger} \mathbf{U}\right]_{i, i}$ with $\mathbf{K} \in$ $\mathbb{R}^{\mu \times \mu}$ a constant matrix whose entries are given by $[\mathbf{K}]_{i, j}=\kappa$ for $i, j=1, \ldots, \mu$.

Proof: See Appendix A.

Lemma 1 provides a closed-form expression for the MGF of $\gamma$. It is easy to prove that, when no correlation is applied, then $\lambda_{i}=1$ and $d_{i}=\kappa$ for $i=1, \ldots, \mu$. If so, then (9) becomes the MGF of the $\kappa-\mu$ shadowed distribution given in [7, eq. (5)]. 


$$
D_{i}\left(k_{1}, \ldots, k_{N-1}\right)=\prod_{t=1}^{\mu} \frac{\left(\frac{\mu(k+1)}{\lambda_{t} \bar{\gamma}}-\beta_{i}\right)^{m-k_{t}-1}}{\Gamma(m)^{-1} \Gamma\left(m-k_{t}\right)} \prod_{r=1}^{i-1} \frac{(-1)^{k_{r+\mu}}\left(q_{r} m\right)_{k_{r+\mu}}}{\left(\beta_{r}-\beta_{i}\right)^{q_{r} m+k_{r+\mu}}} \prod_{l=i+1}^{n} \frac{(-1)^{k_{l+\mu-1}}\left(q_{l} m\right)_{k_{l+\mu-1}}}{\left(\beta_{l}-\beta_{i}\right)^{q_{l} m+k_{l+\mu-1}}} .
$$

From $M_{\gamma}(s)$, we now calculate the PDF and the CDF of $\gamma$. In order to do so, it is necessary to perform some algebraic manipulations in (9). Hence, $M_{\gamma}(s)$ is rewritten in terms of a rational polynomial as follows:

$$
M_{\gamma}(s)=\frac{1}{\prod_{i=1}^{\mu} 1-\frac{\lambda_{i} \bar{\gamma}}{\mu(\kappa+1)} s}\left(\frac{\prod_{j=1}^{\mu} 1-\frac{\lambda_{j} \bar{\gamma}}{\mu(\kappa+1)} s}{P(s)}\right)^{m}
$$

where $P(s)$ is the $\mu$-th order polynomial given by

$$
P(s)=m \prod_{i=1}^{\mu} 1-\frac{\lambda_{i} \bar{\gamma}}{\mu(\kappa+1)} s-\sum_{j=1}^{\mu} \frac{d_{j} \lambda_{j} \bar{\gamma}}{\mu(\kappa+1)} s \prod_{\substack{k=1 \\ k \neq j}}^{\mu} 1-\frac{\lambda_{k} \bar{\gamma}}{\mu(\kappa+1)} s .
$$

Therefore, denoting as $\beta_{i}$ for $i=1, \ldots, n$ the distinct roots of $P(s)$ with multiplicity $q_{i}$, the rational function in (10) is expressed as

$$
M_{\gamma}(s)=\frac{(-\bar{\gamma})^{-\mu} \mu^{\mu}(k+1)^{\mu}}{\left(1+\sum_{k=1}^{\mu} \frac{d_{k}}{m}\right)^{m} \prod_{l=1}^{\mu} \lambda_{l}} \frac{\prod_{j=1}^{\mu}\left(s-\frac{\mu(\kappa+1)}{\lambda_{j} \bar{\gamma}}\right)^{m-1}}{\prod_{i=1}^{n}\left(s-\beta_{i}\right)^{q_{i} m}} .
$$

From (12), the PDF and CDF of $\gamma$ are provided in the following lemmas.

Lemma 2: Let $\gamma \sim \mathcal{C} \mathcal{S}_{\kappa \mu}(\bar{\gamma} ; \kappa, \mu, m, \boldsymbol{\Sigma})$. Then, its PDF is given by

$$
f_{\gamma}(\gamma)=\alpha \sum_{i=1}^{n} \sum_{j=1}^{q_{i} m} A_{i, j} \gamma^{j-1} e^{-\beta_{i} \gamma}
$$

with

$$
\begin{aligned}
\alpha & =\frac{\mu^{\mu}(\kappa+1)^{\mu}}{\bar{\gamma}^{\mu} \Gamma(j)}\left(1+\sum_{j=1}^{\mu} \frac{d_{j}}{m}\right)^{-m} \prod_{l=1}^{\mu} \lambda_{l}^{-1}, \\
A_{i, j} & =\sum_{\substack{k_{1}+\ldots+k_{N-1}=q_{i} m-j \\
k_{1}, \ldots, k_{\mu} \leq m-1}} \frac{1}{\prod_{p=1}^{N-1} k_{p} !} D_{i}\left(k_{1}, \ldots, k_{N-1}\right)
\end{aligned}
$$

where $N=n+\mu$ and $D_{i}\left(k_{1}, \ldots, k_{N}\right)$ is given in (8) at the top of this page. The sum in (12) is over all combinations of $k_{1}, \ldots, k_{N-1}$ that satisfy $\sum_{p=1}^{N-1} k_{p}=q_{i} m-j$ and $k_{1}, \ldots, k_{\mu} \leq m-1$.

Proof: The PDF of $\gamma$ is obtained by means of an inverse Laplace transform as $f_{\gamma}(\gamma)=\mathcal{L}^{-1}\left\{M_{\gamma}(-s)\right\}$. By performing a partial fraction expansion of the rational polynomial in (12), $M_{\gamma}(-s)$ is rewritten as

$$
M_{\gamma}(-s)=\alpha \sum_{i=1}^{n} \sum_{j=1}^{q_{i} m} \frac{A_{i, j}}{\left(s+\beta_{i}\right)^{j}}
$$

where $A_{i, j}$ are the partial fraction expansion residues given in (12) as proved in Appendix B. Finally, (10) is obtained by applying the Laplace transform pair given in [16, eq. (5.4.1)].

Lemma 3: Let $\gamma \sim \mathcal{C} \mathcal{S}_{\kappa \mu}(\bar{\gamma} ; \kappa, \mu, m, \boldsymbol{\Sigma})$. Then, its $\mathrm{CDF}$ is given by

$$
F_{\gamma}(\gamma)=1+\alpha \sum_{i=1}^{n} \sum_{j=1}^{q_{i} m} C_{i, j} \gamma^{j-1} e^{-\beta_{i} \gamma}
$$

with

$$
C_{i, j}=\sum_{\substack{k_{1}+\ldots+k_{N}=q_{i} m-j \\ k_{1}, \ldots, k_{\mu} \leq m-1}} \frac{D_{i}\left(k_{1}, \ldots, k_{N-1}\right) k_{N} !(-1)^{k_{N}}}{\prod_{p=1}^{N} k_{p} !\left(-\beta_{i}\right)^{1+k_{N}}} .
$$

Proof: Following the same steps as in the previous proof, the CDF of $\gamma$ is derived from (12) as $F_{\gamma}(\gamma)=\mathcal{L}^{-1}\left\{\frac{1}{s} M_{\gamma}(s)\right\}$ by performing a partial fraction decomposition and applying [16, eq. (5.4.1)] with $C_{i, j}$ the residues given in (13) as deduced from the proof of $A_{i, j}$ in Appendix B.

Lemmas 2 and 3 provide simple closed-form expressions for the PDF and CDF of $\gamma$ in terms of a finite number of elementary functions, i.e. exponentials and powers. In fact, these expressions have a similar form to those presented in [10] for the PDF and the CDF of the original $\kappa-\mu$ shadowed distribution with integer parameters. This is an important result, since the larger generality introduced in this paper does not come at the price of an increased analytical complexity.

As introduced before, the correlated $\kappa-\mu$ shadowed distribution arises as a generalization of a wide variety of fading models, thus unifying the statistical characterization of all these underlying models. The tractability of the here derived expressions make them useful for further analytical purposes, specially in the case of multivariate distributions (Rayleigh, Rician and Rician shadowed) for which the results are generally scarce due to their mathematical complexity.

Note that, although we here give expressions for the main statistics of the instantaneous SNR, the PDF and the CDF of the signal envelope $R$ are straightforwardly obtained from (10) and (12) as $f_{R}(r)=2 r f_{\gamma}\left(r^{2}\right)$ and $F_{R}(r)=F_{\gamma}\left(r^{2}\right)$, respectively, with $\bar{\gamma}$ being replaced by $\mathbb{E}\left[R^{2}\right]$.

\section{Numerical Results}

We will now provide some numerical results with the aim of exemplifying the influence of the parameters of the newly proposed model over the distribution of the received signal amplitude $R$. Since the novelty of this model is the addition of a correlation between clusters, we will here focus on the analysis of the impact of such correlation matrix $\boldsymbol{\Sigma}$. For the sake of simplicity, we consider an exponential correlation 


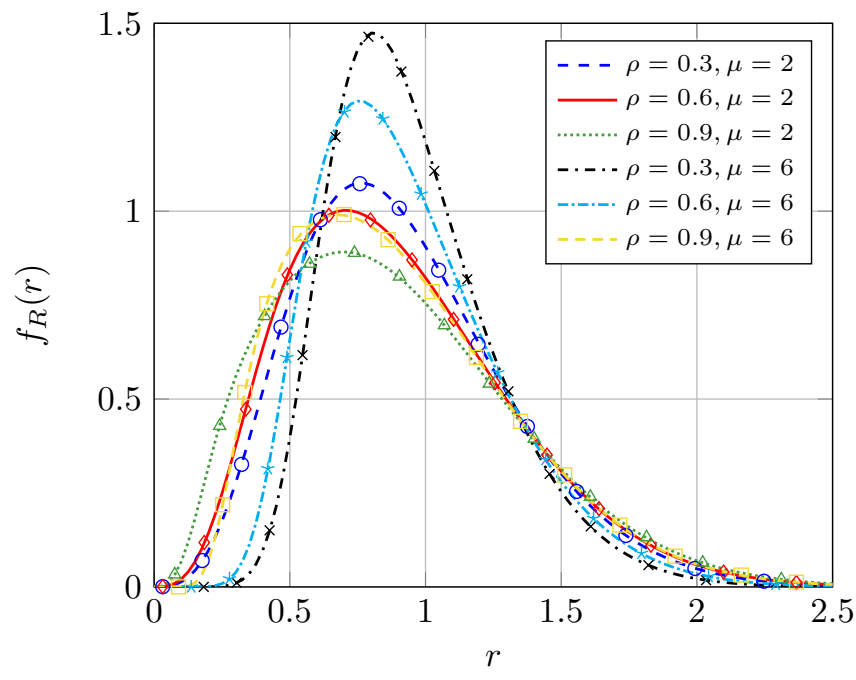

Fig. 1. Signal envelope distribution for different values of $\mu$ and $\rho$ with $\kappa=1, m=1$ and $\mathbb{E}\left[R^{2}\right]=1$. Solid lines correspond to the exact PDF while markers correspond to Monte Carlo simulations.

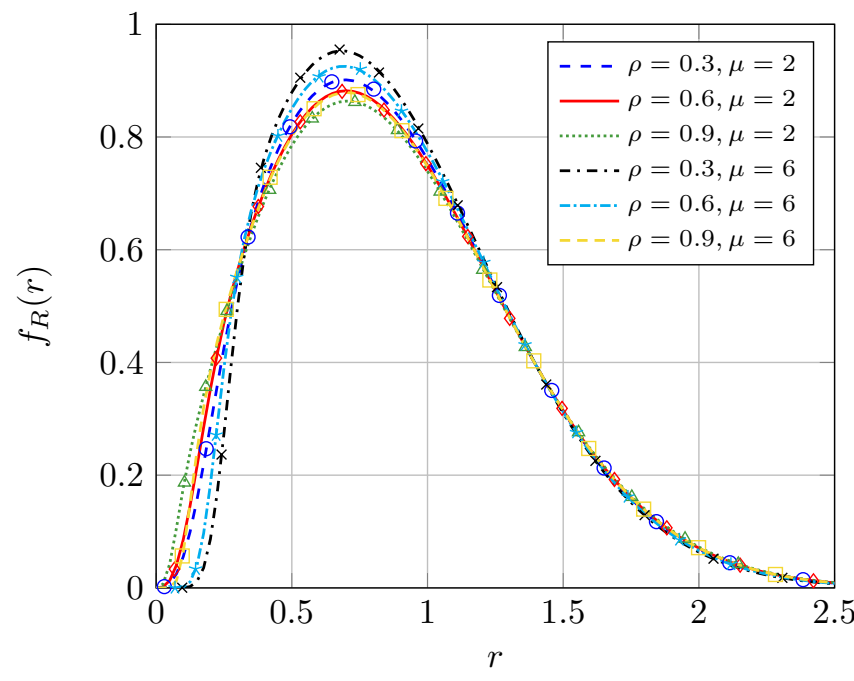

Fig. 2. Signal envelope distribution for different values of $\mu$ and $\rho$ with $\kappa=10, m=1$ and $\mathbb{E}\left[R^{2}\right]=1$. Solid lines correspond to the exact PDF while markers correspond to Monte Carlo simulations.

matrix whose entries are given by $[\boldsymbol{\Sigma}]_{i, j}=\rho^{|i-j|}$ with $0 \leq|\rho|<1$, where a value of $\rho=0$ implies uncorrelated scattered components.

Thus, we show in Figs. 1 and 2 the PDF of the received signal envelope for different values of $\rho$ and $\mu$ in a weak $(\kappa=1)$ and a strong $(\kappa=10)$ LoS scenario, respectively. The value of $m$ is fixed to $m=1$, corresponding to the case of heavy random fluctuation of the dominant component of each cluster. Regarding both figures, it is easy to notice that the influence of $\rho$ is less relevant for large $\kappa$. This is a coherent result, since the correlation factor only affects to the scattered component (NLoS) according to the physical model in (1). We also observe in Fig. 1 that low values of $\rho$ render more sparse values of the signal amplitude. Besides, the effect of

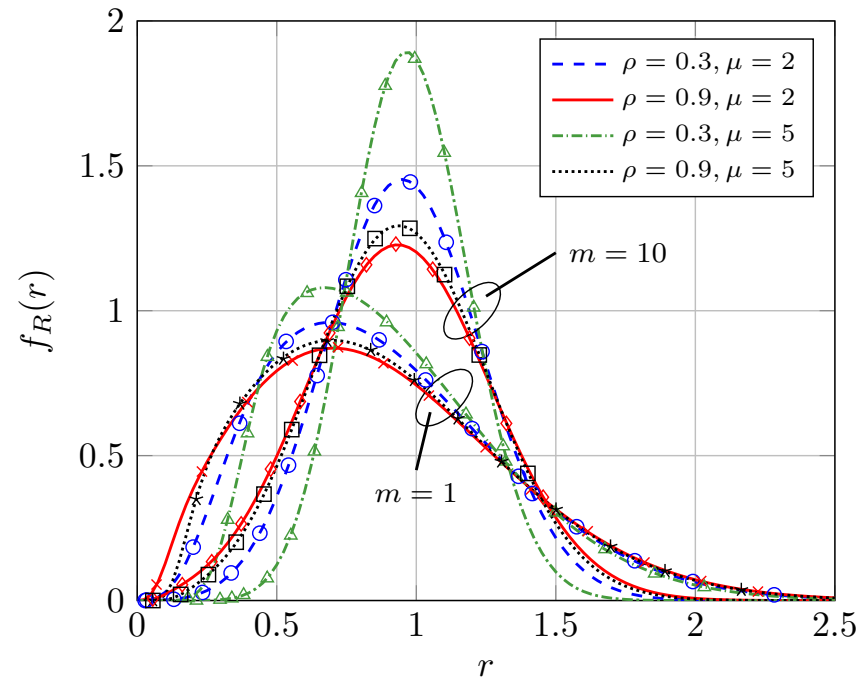

Fig. 3. Signal envelope distribution for different values of $\mu, \rho$ and $m$ with $\kappa=4$ and $\mathbb{E}\left[R^{2}\right]=1$. Solid lines correspond to the exact PDF while markers correspond to Monte Carlo simulations.

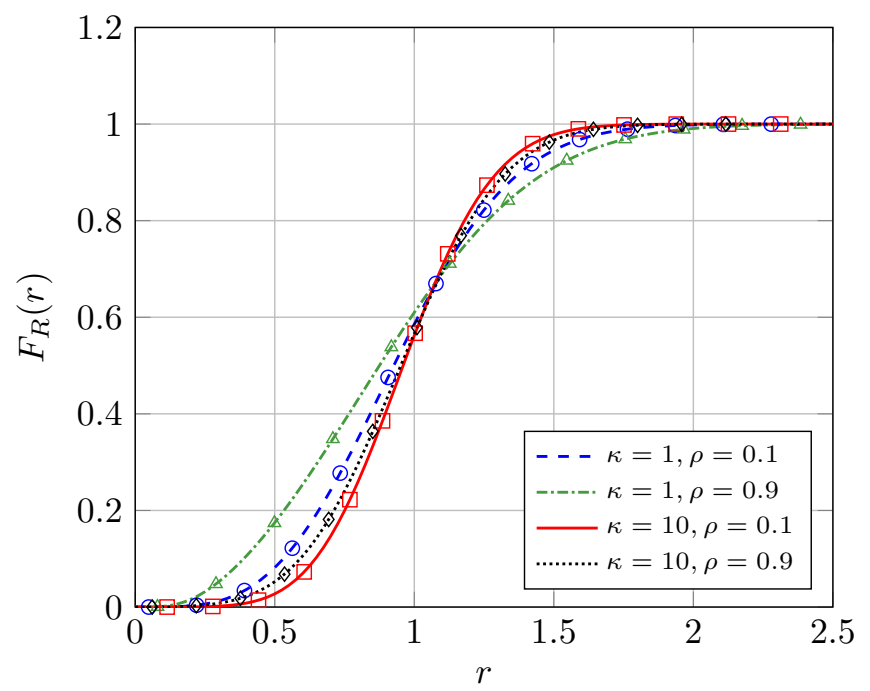

Fig. 4. CDF of the signal envelope for different values of $\kappa$ and $\rho$ with $\mu=2, m=5$ and $\mathbb{E}\left[R^{2}\right]=1$. Solid lines correspond to the exact PDF while markers correspond to Monte Carlo simulations.

that correlation has a larger impact as $\mu$ grows, which seems to be logical since we are increasing the number of clusters.

Fig. 3 depicts the PDF of the received signal amplitude for different values of $\rho, \mu$ and $m$ for a fixed LoS power. We compare the effect of $m$ in the cases of low correlation ( $\rho=$ $0.3)$ and high correlation $(\rho=0.9)$. Nonetheless, the impact of the correlation factor seems to be independent of the value of $m$. However, a severe fluctuation of the dominant component $(m=1)$ is more detrimental for the signal envelope, as we already saw in the original model $[7,10]$. Finally, we represent in Fig. 4 the shape of the CDF of $R$ in a weak $(\kappa=1)$ and a strong $(\kappa=10)$ for low and high intercluster correlation. We fix $\mu=2$ and $m=5$ in order to minimize the influence of the 
LoS fluctuation, observing that low values of $\kappa$ and $\rho$ makes the slope of the CDF rise slowly, as expected from the results of the previous figures.

\section{CONCLUSIONS}

We here presented the correlated $\kappa-\mu$ shadowed fading model, which arises as a generalization of the original $\kappa-\mu$ shadowed distribution. This new model considers an arbitrary correlation between the scattered components of each cluster, represented by the matrix parameter $\boldsymbol{\Sigma}$. The MGF, PDF and $\mathrm{CDF}$ of the proposed model are given in closed-form in terms of elementary functions, being appropriated for further theoretical calculations and providing a simple framework for the analysis of the underlying fading models. The correlated $\kappa-\mu$ shadowed statistics are also useful for characterizing the multivariate Rayleigh, Rician and Rician shadowed distributions, which are particular cases of the here proposed model.

The influence of the arbitrary correlation is of relevant interest specially in weak LoS scenarios, rendering more sparse values of the fading amplitude as the correlation factor increases. This effects remains even vanishing the fluctuation of the dominant component.

\section{APPENDIX A \\ PROOF OF LEMMA 1}

Let us consider the physical model in (5). When conditioned on $\xi$, the received signal power can be seen as the summation of $\mu$ independent and non-identically distributed Rician random variables whose MGFs are given in [1, eq. (2.17)] by setting $n^{2}=\xi^{2}\left|\widetilde{p}_{i}\right|^{2} / \sigma^{2}$ and $\bar{\gamma}=\lambda_{i}\left(\xi^{2}\left|\widetilde{p}_{i}\right|^{2}+\sigma^{2}\right)$ for $i=1, \ldots, \mu$. As such, the conditional MGF of $W$ is obtained as the product of the MGFs of each Rician variable in (5). By performing some algebraic manipulations, the conditional MGF of the the instantaneous SNR $\gamma$ is given as

$$
M_{\left.\gamma\right|_{\xi}}(s)=\mu^{\mu}(\kappa+1)^{\mu} \prod_{i=1}^{\mu} \frac{\exp \left(\frac{\xi^{2} d_{i} \lambda_{i} \bar{\gamma} s}{\mu(\kappa+1)-\lambda_{i} \bar{\gamma} s}\right)}{\mu(\kappa+1)-\lambda_{i} \bar{\gamma} s}
$$

Finally, the unconditional MGF of $\gamma$ is obtained by averaging (14) as

$$
M_{\gamma}(s)=\int_{0}^{\infty} M_{\gamma \mid \xi}(s) f_{\xi^{2}}(x) d x
$$

with $f_{\xi^{2}}(x)$ the PDF of the Gamma distribution, yielding (9).

\section{APPENDIX B}

\section{DERIVATION OF PARTIAL FRACTION EXPANSION RESIDUES}

Residues $A_{i, j}$ arise from performing a partial fraction expansion in (12) after evaluating $M_{\gamma}(-s)$. Then, $A_{i, j}$ for $i=1, \ldots, n$ and $j=1, \ldots, q_{i} m$ are defined as

$$
A_{i, j}=\left.\frac{1}{\left(\widetilde{q}_{i, j}\right) !} \frac{d^{\widetilde{q}_{i, j}}}{d s^{\widetilde{q}_{i, j}}}\left(\frac{\prod_{l=1}^{\mu}\left(s+\frac{\mu(\kappa+1)}{\lambda_{l} \bar{\gamma}}\right)^{m-1}}{\prod_{k=1, k \neq i}^{n}\left(s+\beta_{k}\right)^{q_{k} m}}\right)\right|_{s=-\beta_{i}}
$$

with $\widetilde{q}_{i, j}=q_{i} m-j$. By applying the generalization of Léibniz's rule, the derivatives in (16) can be expressed as a linear combination of the derivatives of the distinct binomials. Therefore, since the $k$-th derivative of a binomial is a wellknown expression which is given by

$$
\frac{d^{q}}{d x^{q}}(x+a)^{\nu}= \begin{cases}\frac{(-1)^{q}(-\nu)_{q}}{(x+a)^{-\nu+q}} & \text { if } \nu<0 \\ \frac{\nu !}{(\nu-q) !}(x+a)^{\nu-q} & \text { if } \nu>0\end{cases}
$$

the final expression for $A_{i, j}$ is obtained by applying (17) in (16), yielding (12). By following the same steps as with $A_{i, j}$, we obtain (13) as the residues resulting from performing a partial fraction expansion of $M_{\gamma}(-s) / s$.

\section{REFERENCES}

[1] M. K. Simon and M.-S. Alouini, Digital Communication over Fading Channels. John Wiley \& Sons, 2005, vol. 95.

[2] M. D. Yacoub, "The $\kappa-\mu$ distribution and the $\eta-\mu$ distribution," IEEE Antennas and Propag. Mag., vol. 49, no. 1, pp. 68-81, Feb. 2007.

[3] P. C. Sofotasios, E. Rebeiz, L. Zhang, T. A. Tsiftsis, D. Cabric, and S. Freear, "Energy detection based spectrum sensing over $\kappa-\mu$ and $\kappa-\mu$ extreme fading channels," IEEE Trans. Veh. Technol., vol. 62, no. 3, pp. 1031-1040, Mar. 2013.

[4] S. L. Cotton, W. G. Scanlon, and J. Guy, "The $\kappa-\mu$ distribution applied to the analysis of fading in body to body communication channels for fire and rescue personnel," IEEE Antennas Wireless Propag. Lett., vol. 7 pp. 66-69, 2008.

[5] J. Zhang, Z. Tan, H. Wang, Q. Huang, and L. Hanzo, "The effective throughput of MISO systems over $\kappa-\mu$ fading channels," IEEE Trans. Veh. Technol., vol. 63, no. 2, pp. 943-947, Feb. 2014.

[6] M. D. Yacoub, "The $\alpha-\eta-\kappa-\mu$ fading model," IEEE Transactions on Antennas and Propagation, vol. 64, no. 8, pp. 3597-3610, Aug. 2016.

[7] J. F. Paris, "Statistical characterization of $\kappa-\mu$ shadowed fading," IEEE Trans. Veh. Technol., vol. 63, no. 2, pp. 518-526, Feb. 2014.

[8] A. Abdi, W. C. Lau, M. S. Alouini, and M. Kaveh, "A new simple model for land mobile satellite channels: first- and second-order statistics," IEEE Transactions on Wireless Communications, vol. 2, no. 3, pp. 519528, May 2003.

[9] M. R. Bhatnagar, "On the sum of correlated squared $\kappa-\mu$ shadowed random variables and its application to performance analysis of MRC," IEEE Trans. on Veh. Technol., vol. 64, no. 6, pp. 2678-2684, June 2015.

[10] F. J. Lopez-Martinez, J. F. Paris, and J. M. Romero-Jerez, "The $\kappa$ - $\mu$ shadowed fading model with integer fading parameters," IEEE Trans. Veh. Technol., vol. 66, no. 9, pp. 7653-7662, Sept. 2017.

[11] J. R. Mendes and M. D. Yacoub, "A general bivariate Ricean model and its statistics," IEEE Transactions on Vehicular Technology, vol. 56 no. 2, pp. 404-415, Mar. 2007.

[12] N. C. Beaulieu and K. T. Hemachandra, "Novel simple representations for Gaussian class multivariate distributions with generalized correlation," IEEE Transactions on Information Theory, vol. 57, no. 12, pp. 8072-8083, Dec. 2011.

[13] Y. Chen and C. Tellambura, "Infinite series representations of the trivariate and quadrivariate Rayleigh distribution and their applications," IEEE Transactions on Communications, vol. 53, no. 12, pp. 2092-2101, Dec 2005.

[14] J. Lopez-Fernandez, J. F. Paris, and E. Martos-Naya, "Bivariate Rician shadowed fading model," IEEE Transactions on Vehicular Technology, vol. 67, no. 1, pp. 378-384, Jan 2018.

[15] R. Horn and C. Johnson, Matrix Analysis. Cambridge University Press, 1990.

[16] A. Erdelyi, W. Magnus, F. Oberhettinger, and F. G. Tricomi, Tables of Integral Transforms: Vol.: 1. McGraw-Hill Book Company, Incorporated, 1954 